\title{
Characteristics of surface-wave and volume-wave plasmas produced with internally mounted large-area planar microwave launcher
}

\author{
Masaaki Nagatsu, ${ }^{a)}$ Katsutoshi Naito, Akihisa Ogino, and Keigo Ninomiya \\ Graduate School of Science and Engineering, Shizuoka University, 3-5-1 Johoku, \\ Hamamatsu 432-8561, Japan \\ Shohei Nanko \\ Nissin Inc., 10-7 Kamei-cho, Takarazuka 665-0047, Japan
}

(Received 13 June 2005; accepted 27 August 2005; published online 14 October 2005)

\begin{abstract}
We studied discharge characteristics of microwave plasmas excited with a large-area planar microwave launcher installed internally in a $600-\mathrm{mm}$-diam cylindrical vacuum chamber. With the microwave power less than roughly $400 \mathrm{~W}$, we demonstrated the large volumetric volume-wave plasma (VWP) spread in the entire chamber at a pressure of 14-27 Pa in He. Above $400 \mathrm{~W}$, the plasma discharge made a sudden transition to higher-density, uniform surface-wave plasma (SWP) having a spatial uniformity of $\pm 3.5 \%$ over $300 \mathrm{~mm}$ in diameter. Electron energy probability functions in the downstream region were studied using Langmuir probe measurements with Druyvesteyn method in both the SWP and VWP discharges. (c) 2005 American Institute of Physics.
\end{abstract}

[DOI: $10.1063 / 1.2108119$ ]

Large-area plasma sources are indispensable for largesized ultralarge-scale integration (ULSI) fabrication with nanosized gate widths or meter-sized liquid-crystal display panel manufacturing. In various plasma processes required in fabricating the electronic devices, such as etching, ashing, or plasma chemical-vapor deposition (CVD), spatial uniformity of the plasma source was one of the important requirements, in addition to the demand of high-density plasma. Furthermore, in the fabrication of amorphous or polycrystalline silicon films, nanocrystalline diamond films or carbon nanotubes, for instance, the large-area high-density plasma sources have also been needed. Moreover, application of large volumetric plasma to medical sterilization has been recently noticed.

Among the various plasma devices, surface-wave plasma (SWP) excited by microwave is one of the promising plasma sources satisfying the requirements for a large-area plasma processing tool. ${ }^{1-10}$ The advantages of SWP are that the high-density plasma can be produced even at low pressure, say, about $1 \mathrm{~Pa}$, and large-area uniform plasma can be produced using a slot antenna technique. ${ }^{2,3,5-10}$ In the SWP, it was found that density jumps have occurred when the microwave power or the feedstock gas pressure was changed. They originated from the surface-wave modes determined from the geometrical boundary conditions including the dielectric window and plasma at a given incident wave frequency. ${ }^{11}$ From the practical standpoints, such a discontinuous behavior of the plasma density is undesirable for the actual material processing. Another big issue in enlarging the plasma device is on the dielectric window needed for vacuum sealing. To hold a huge mechanical force, typically 10 tons $/ 1 \mathrm{~m}^{2}$ of dielectric window, one needs a very expensive, thick dielectric plate. Recently we have proposed the cavity type of planar microwave launcher installed inside the chamber to solve the problems on the density jumps and dielectric window. In the proposed microwave launcher system, it is a great advantage that we can use a thinner quartz plate as vacuum window. Moreover, with the present launcher, we

${ }^{a)}$ Electronic mail: tmnagat@ipc.shizuoka.ac.jp can also produce volume-wave plasma (VWP), which has electron density lower than the cutoff density. ${ }^{12}$

In this letter, we present the experimental results on discharge transition between SWP and VWP excited with a large-area planar microwave launcher installed internally in a cylindrical vacuum chamber with $600 \mathrm{~mm}$ in diameter. ${ }^{13}$ Spatial distributions of electron density were measured with a Langmuir probe for both the SWP and VWP. Finite difference time domain (FDTD) method was used to analyze the field intensity distribution radiated from the planar microwave launcher to understand how the plasma was produced. For the applications of the SWP or VWP to etching, plasma CVD, or plasma sterilization, electron energy probability function (EEPF) is one of the important parameters to discuss those performances. Hence, we carried out to measure the EEPF using the Druyvesteyn technique of Langmuir probe measurements with double-frequency modulation method. ${ }^{14,15}$

A schematic drawing of the experimental setup with a microwave launcher is shown in Fig. 1. The cylindrical vacuum chamber with $600 \mathrm{~mm}$ in inner diameter and $350 \mathrm{~mm}$ in height was pumped down to roughly $1 \mathrm{~Pa}$ by a rotary pump. The $2.45 \mathrm{GHz}$ microwave guided by a rectan-

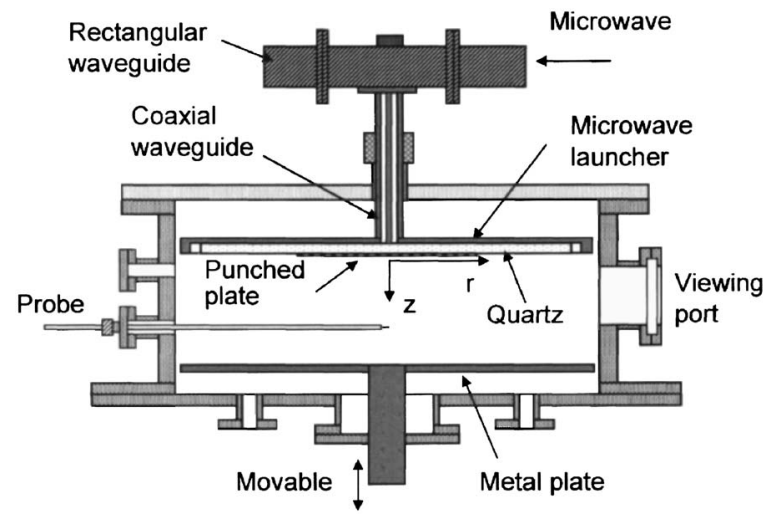

FIG. 1. Schematic drawing of the experimental setup with a planar microwave launcher. 


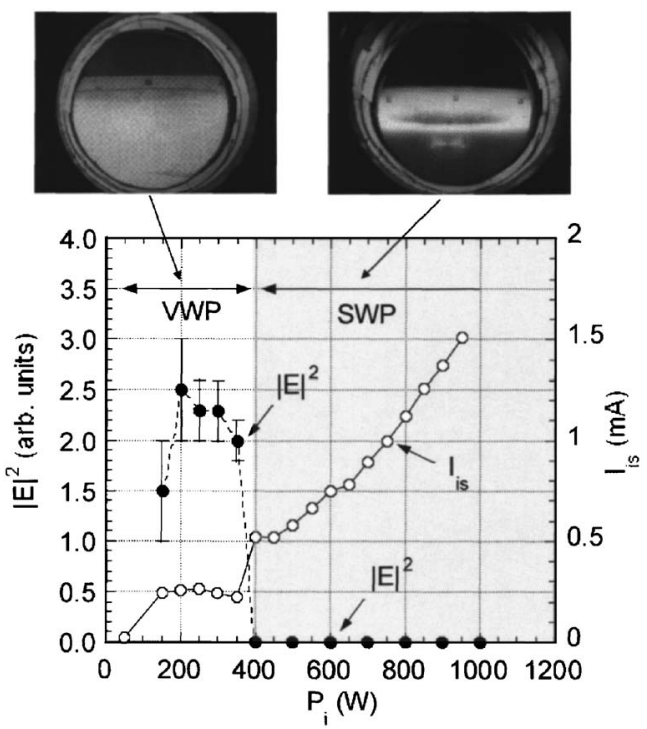

FIG. 2. Incident power dependencies of ion saturation currents and electricfield intensity in He plasma discharges at a pressure of 18.6 Pa. Photographs show VWP at an incident power of $200 \mathrm{~W}$ (left) and SWP at an incident power $700 \mathrm{~W}$ (right).

gular waveguide was transferred to the microwave launcher via a coaxial waveguide. Microwave power of $2.45 \mathrm{GHz}$ magnetron is variable from zero to $1.5 \mathrm{~kW}$. In the present microwave launcher, the quartz plate with $420 \mathrm{~mm}$ in diameter and $15 \mathrm{~mm}$ in thickness was used. For the spatial distribution measurements of ion saturation currents and electricfield intensity, we used a Langmuir probe with a 4-mm-length, 0.7-mm-diam platinum wire tip. The probe was shielded by a stainless-steel pipe of $6 \mathrm{~mm}$ in outer diameter, excluding the wire tip. For the measurement of microwave field intensity, the electric fields were picked up locally by the probe wire tip. The output of probe was connected to a spectrum analyzer to measure the power spectrum of detected microwave fields. ${ }^{4}$ The EEPFs were also measured using the Druyvesteyn method of probe measurements with double-frequency modulations at 8 and $10 \mathrm{kHz} .^{16}$

Figure 2 shows the incident power dependencies of ion saturation currents and electric-field intensity in He plasma discharges, where microwave power was varied from $200 \mathrm{~W}$ to $1.0 \mathrm{~kW}$ at a gas flow rate of $100 \mathrm{SCCM}$ (standard cubic centimeter per minute) and a pressure of 18.6 Pa. We observed a discharge transition between volume-wave- and

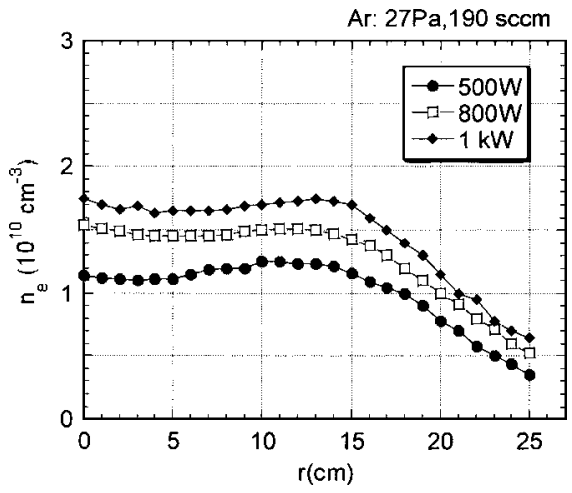

FIG. 3. Spatial distributions of electron density at the axial position of $z=10.5 \mathrm{~cm}$, where the incident power were fixed at $500 \mathrm{~W}, 800 \mathrm{~W}$, and $1 \mathrm{~kW}$, respectively. kW, respectively.
Downloaded 10 Jul 2008 to 133.70.80.50. Redistribution subject to AIP license or copyright; see http://apl.aip

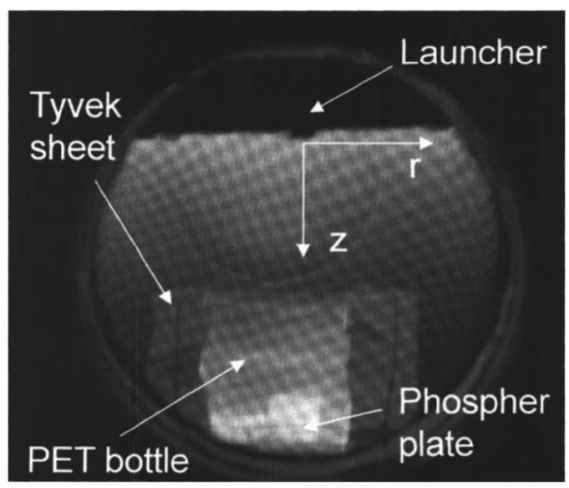

FIG. 4. Photograph of VWP discharge produced inside the PET bottle wrapped by Tyvek sheet.

surface-wave-sustained He plasma discharges at an input power of roughly $400 \mathrm{~W}$ at a pressure of 14-27 Pa. Here, the Langmuir probe was fixed at $r=0$ and $z=10.5 \mathrm{~cm}$ below the microwave launcher. When the microwave power was around $200 \mathrm{~W}$, the plasma discharge entirely spread in the chamber, as shown in Fig. 2. Electric fields were observed inside the chamber, when power was less than $400 \mathrm{~W}$. It is noticed here that the plasma discharges sustained by the volume wave is of underdense, since the peak electron density in VWP is less than the cutoff density, $7.4 \times 10^{10} \mathrm{~cm}^{-3}$. When the incident power increased to $400 \mathrm{~W}$ or more, plasma discharge made a sharp transition from VWP to SWP, where the bright plasma discharge was observed just below the quartz plate of the microwave launcher, as shown in Fig. 2. It is noted that electron density in SWP increases almost linearly with the incident power without any density jumps after the mode transition. In the SWP discharge mode, spatial distributions of electron density were measured by scanning the Langmuir probe horizontally at the axial position of $z$ $=10.5 \mathrm{~cm}$ away from the launcher surface when the incident powers were $500 \mathrm{~W}, 800 \mathrm{~W}$, and $1 \mathrm{~kW}$, respectively. In the downstream region at $z=10.5 \mathrm{~cm}$, we observed considerably uniform density profiles having roughly 1.0-2.0

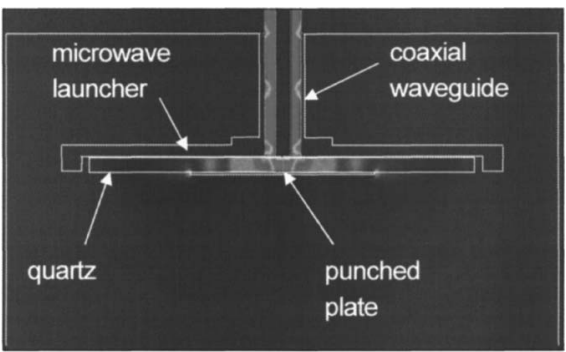

(a)

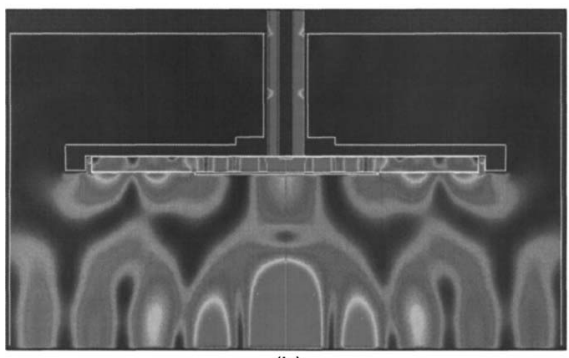

(b)

FIG. 5. Field intensity distributions of (a) 3000 and (b) 40000 calculation

AIP license or copyright; see http://apl.aip.org/apl/copyright.jsp 


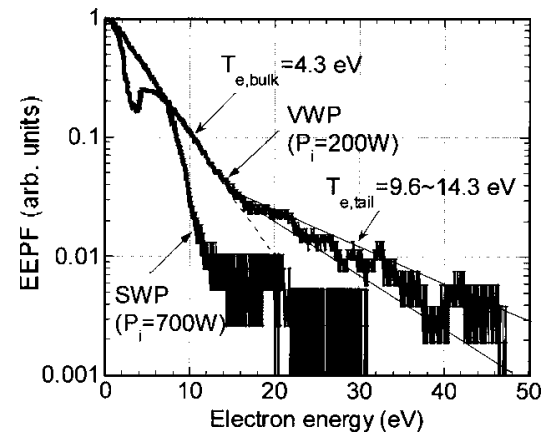

FIG. 6. Comparison of electron energy probability functions in He plasma discharges of VWP at an incident power of $200 \mathrm{~W}$ and SWP at an incident power of $700 \mathrm{~W}$.

$\times 10^{11} \mathrm{~cm}^{-3}$ over a diameter of $300 \mathrm{~mm}$ with a spatial uniformity of $\pm 3.5 \%$, as shown in Fig. 3. In the case of the VWP discharge, plasma spreads over the chamber volume. With the VWP discharge mode, we have succeeded to produce the plasma inside the polyethylene terephthalate (PET) bottle wrapped by Tyvek sheet, as shown in Fig. 4. This result suggests that the VWP can be utilized for inner plasma treatment, such as coating or sterilization.

Here, we briefly present the FDTD numerical results of electric-field intensity radiated from the microwave launcher. Figure 5 shows the calculation results of field intensity distributions, where assuming the axial symmetry, we ran the FDTD code with a time step of $0.4 \mathrm{ps}$ and a cell size of $2 \mathrm{~mm}^{12}$ Figure 5(a) shows the calculation result at 3000 steps where microwave has just radiated from the quartz surface toward the chamber. We can expect that the plasma discharge will be produced near quartz surface as the SWP mode, if the microwave field strength is strong enough to produce the discharge at certain pressure. However, if the incident microwave power is not high enough, then microwave will propagate into the chamber and form a certain volume-wave mode structure, as shown in Fig. 5(b). In this case, plasma will be produced in the midspace where the microwave intensities are high enough to ionize the gas. This results in the production of VWP. At a fixed microwave power, the same thing will happen by changing gas pressure. These expectations are consistent with the present experimental results shown in Fig. 2.

Lastly, we discussed the EEPFs in both the plasmas to investigate the plasma production characteristics. In the SWP mode, the electric field was localized near the microwave launcher and strongly damped with a decay length of several millimeters to $1 \mathrm{~cm}^{7}$ Near the quartz window, there exist high-energy components, which might be originated from the interaction between the launched microwave and plasma. ${ }^{16}$ In the downstream region of SWP, however, it is considered that the electron components will be lost by the inelastic collision with neutrals. In Fig. 6, we plotted normalized EEPFs measured at $z=10.5 \mathrm{~cm}$ in the cases of SWP and VWP, where the incident microwave power was fixed at $200 \mathrm{~W}$ in VWP and $700 \mathrm{~W}$ in SWP. In the case of SWP, especially, strong dips in EEPF are seen in the electron energy ranges of $2-6 \mathrm{eV}$ and higher than $8 \mathrm{eV}$. It is speculated that such dips in the lower-energy regions were stemmed from the energy loss due to excitation of neutrals, such as residual oxygen atoms or molecules, since there was no electric field in the downstream region in SWP.
On the other hand, the EEPF in the VWP indicates a bi-Maxwellian with an electron bulk temperature of $4.3 \mathrm{eV}$ and high energetic components of 9.6-14.3 eV. It is deduced that these energetic components might be created by collisional heating by the volume-wave electric fields. From optical emission measurements, it was also found that the relative intensities of He II lines in emission spectrum of VWP were strongly enhanced compared to those of SWP. This result also sustains that VWP had a larger content of energetic electrons in EEPF than SWP.

In conclusion, we presented experimental results on the He plasma discharges sustained by surface wave and volume wave excited with a large-area planar microwave launcher installed internally in a 600 -mm-diam cylindrical vacuum chamber. We presented mode transition between SWP and VWP by changing the incident microwave power. In the SWP discharge, we observed uniform density profile over $300 \mathrm{~mm}$ in diameter and the density changed linearly with the incident microwave power without any density jumps. This result suggests that the present launcher can be utilized as module unit in realizing meter-sized plasma source. Furthermore, the electron energy probability functions were investigated for both the plasma discharges. In the downstream region of SWP, the high-energy components are lost via neutral collision, so that the low-temperature processing will be realized. In the VWP, bi-Maxwellian electron distribution function was observed. Therefore, VWP will be also useful in the material processing, such as internal coating or sterilization of plastic bags and plasma CVD on the isolated heater stage.

This work has been supported in part by the Grants-inAid for Scientific Research and performed under the 21st Century COE Program "Research and Education Center of Nanovision Science" by the Japan Society for the Promotion of Science.

${ }^{1}$ K. Komachi and S. Kobayashi, J. Microwave Power Electromagn. Energy 25, 236 (1990).

${ }^{2}$ G. Sauve, M. Moisan, and Z. Zakrzewski, J. Microwave Power Electromagn. Energy 28, 123 (1993).

${ }^{3}$ F. Werner, D. Korzec, and J. Engemann, Plasma Sources Sci. Technol. 3, 473 (1994).

${ }^{4}$ E. Bluem, S. Bechu, C. Boisse-Laporte, P. Leprince, and J. Marec, J. Phys. D 28, 1529 (1995).

${ }^{5}$ M. Nagatsu, G. Xu, M. Yamage, M. Kanoh, and H. Sugai, Jpn. J. Appl. Phys., Part 2 35, L341 (1996).

${ }^{6}$ M. Nagatsu, G. Xu, I. Ghanashev, M. Kanoh, and H. Sugai, Plasma Sources Sci. Technol. 6, 427 (1997).

${ }^{7}$ M. Nagatsu, I. Ghanashev, and H. Sugai, Plasma Sources Sci. Technol. 7, 230 (1998).

${ }^{8}$ H. Sugai, I. Ghanashev, and M. Nagatsu, Plasma Sources Sci. Technol. 7, 192 (1998)

${ }^{9}$ M. Nagatsu, A. Ito, N. Toyoda, and H. Sugai, Jpn. J. Appl. Phys., Part 2 38, L679 (1999).

${ }^{10}$ I. Odrobina, J. Kudela, and M. Kando, Plasma Sources Sci. Technol. 7, 238 (1998)

${ }^{11}$ I. Ghanashev, M. Nagatsu, and H. Sugai, Jpn. J. Appl. Phys., Part 1 36, 337 (1997).

${ }^{12}$ A. Ogino, K. Naito, F. Terashita, S. Nanko, and M. Nagatsu, Jpn. J. Appl. Phys., Part 2 44, L352 (2005).

${ }^{13}$ M. Nagatsu, K. Naito, A. Ogino, and S. Nanko, Plasma Sources Sci. Technol. (submitted).

${ }^{14}$ U. Kortshagen and H. Schlüter, J. Phys. D 24, 1585 (1991).

${ }^{15}$ U. Kortshagen, Microwave Discharges: Fundamentals and Applications, edited by C. M. Ferreira and M. Moisan (Plenum, New York, 1993), p. 303.

${ }^{16}$ M. Nagatsu, T. Niwa, and H. Sugai, Appl. Phys. Lett. 81, 1966 (2002). 